\title{
Kryoextraktion bei unterschiedlichen Formen einer Atemwegsobstruktion: Vier exemplarische Fälle
}

\author{
Use of Cryoextraction in Different Types of Airway Obstruction
}

Autoren

Institute
K.-J. Franke, G. Nilius, K.-H. Rühle

Klinik für Pneumologie und Beatmungsmedizin, Helios Klinik Ambrock, Universität Witten/Herdecke eingereicht 28.1.2010

akzeptiert 11.2.2010

\section{Bibliografie}

Dol http://dx.doi.org/ 10.1055/s-0029-1244046

Online-Publikation: 13. 4. 2010

Pneumologie 2010; 64:

387-389 @ Georg Thieme

Verlag KG Stuttgart · New York

ISSN 0934-8387

Korrespondenzadresse Dr. Karl-Josef Franke Klinik für Pneumologie und Beatmungsmedizin, Helios Klinik Ambrock, Universität Witten/Herdecke Ambrocker Weg 60 58091 Hagen karl-josef.franke@helioskliniken.de

\section{Zusammenfassung \\ $\nabla$}

Durch die Entwicklung mechanisch stabiler Kryosonden ist eine direkte endoskopische Entfernung von Gewebe aus dem Bronchialsystem mittels Kältetechnik möglich geworden. Das Gewebe wird an der Sondenspitze angefroren und durch Zug extrahiert, wobei die Sonde zusammen mit dem Bronchoskop entfernt werden muss. Dieses auch als Kryoextraktion bezeichnete Verfahren liefert zudem Biopsate, die histopathologisch gut beurteilbar sind. Wir berichten exemplarisch über vier Patienten mit unterschiedlichen Formen der Atemwegsverlegung, bei denen zur raschen Rekanalisation eine Kryoextraktion durchgeführt wurde. Neben einem Soforteffekt bietet die Methode weitere Vorteile: Es besteht keine Notwendigkeit einer Clean-up-Bronchoskopie, das Verfahren ist komplikationsarm und kostengünstig. Die Indikationen waren eine mucoid impaction bei allergischer bronchopulmonaler Aspergillose, eine Fremdkörperaspiration, die Entfernung subglottischen Granulationsgewebes vor geplanter Trachealkanülenentfernung und eine Verlegung von Trachea und Hauptbronchien durch ein Karzinosarkom. Im letztgenannten Fall konnte außerdem die Diagnose anhand der Kryobiopsate gestellt werden.

\section{Einleitung}

$\nabla$

Es sollen im Folgenden beispielhaft verschiedene Indikationen für den Einsatz der Kryoextraktion zur raschen Atemwegsrekanalisation präsentiert werden. Andere Verfahren mit Soforteffekt wie Laser, Argon-Plasma-Koagulation, Schlinge, Microdebrider, Zange oder Absaugung verfügen über ein schmaleres Indikationsspektrum. Es wird zudem auf die hohe diagnostische Wertigkeit der Kryobiopsie hingewiesen.

\section{Abstract \\ $\nabla$}

With the development of mechanically stable cryoprobes, direct endoscopic removal of tissue from the bronchial system with cryotherapy is now possible. Tissue is frozen to the tip of the probe and removed by pulling on the probe together with the fiberscope. This procedure, also referred to as cryoextraction, additionally provides good quality biopsy specimens for histopathological examination. We report here on four patients with different forms of bronchial obstruction, in whom cryoextraction was performed for rapid recanalization. In addition to the immediate effect, the procedure has few complications and is costeffective. Indications were mucoid impaction, foreign body aspiration, removal of suprastomal granulation tissue prior to decannulation after prolonged mechanical ventilation, and obstruction of the trachea and the main bronchi by a carcinosarcoma. In the latter case, the diagnosis was additionally based on a specimen obtained by cryobiopsy.

\section{Fall 1 \\ $\nabla$}

Eine 71-jährige Frau mit allergischer bronchopulmonaler Aspergillose wurde mit Dyspnoe, einer Hypoxämie und radiologisch einer subtotalen linksseitigen Atelektase vorgestellt. Bronchoskopisch wurde über das starre Bronchoskop das flexible Bronchoskop vorgeschoben. Das linke Bronchialsystem war bis in den Hauptbronchus hinein mit grauem, kautschukartigem Sekret ausgekleidet, das auch durch Einführen eines großlumigen Absaugschlauches nicht zu mobilisieren war. Über den Arbeitskanal des Fiberskops wurde eine 


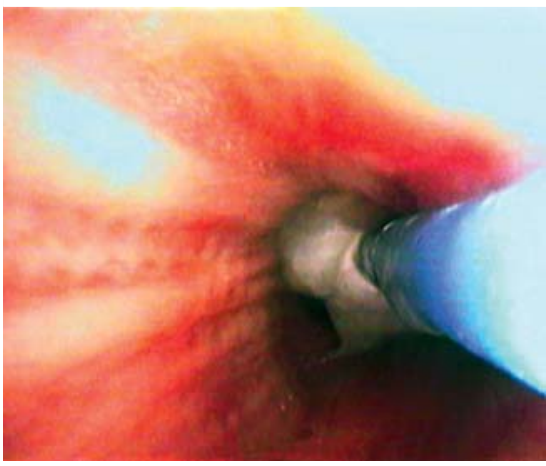

Abb. 1 Linker Hauptbronchus. Anhaften von Mucus am distalen Ende der Kryosonde. Zur Kälteapplikation wird mit hohem Druck $\mathrm{CO}_{2}$ durch den zentralen Gaskanal der Sonde gepresst. Beim Austritt des Gases an der Sondenspitze entstehen Temperaturen bis zu $-50^{\circ} \mathrm{C}$.

Kryosonde mit einem Durchmesser von 2,4 mm vorgeschoben und innerhalb von 3-5 Minuten konnten die bolusartigen Schleimkondensate im Sinne einer mucoid impaction in mehreren Einzelschritten extrahiert werden. Dabei wurde folgende Technik angewandt: Die Sondenspitze wurde in den Mukussee getaucht, unter Kälteapplikation hin und her bewegt und mit dem anhaftenden Schleimballen zusammen mit dem flexiblen Bronchoskop entfernt ( $\bullet$ Abb. 1). Im Verlauf der folgenden Wochen war unter Kortikosteroid-Therapie keine endoskopische Intervention mehr notwendig.

\section{Fall 2}

$\nabla$

Ein 76 Jahre alter Mann, der einen seit mehr als 4 Wochen bestehenden Husten beklagte, zeigte im endoskopischen Bild einen im rechten Hauptbronchus befindlichen Fremdkörper sowie ausgeprägt entzündliche Schleimhautveränderungen. Im Rahmen der durchgeführten starren Bronchoskopie misslang ein Greifen mit der Fremdkörperfasszange, weil die Branchen der Zange nicht zwischen Fremdkörper und Bronchialwand plaziert werden konnten. Nach Vorspiegeln mit dem Fiberskop wurde das distale Ende der flexiblen Kältesonde in Kontakt mit dem Fremdkörper gebracht, dieser angefroren und mobilisiert. Aufgrund seiner Größe konnte der Fremdkörper nicht in das starre Bronchoskop (Innendurchmesser 12,0 mm) hineingezogen werden, eine Entfernung durch die Stimmritze zusammen mit diesem war aber problemlos möglich. Histologisch handelte es sich um ein teilweise nekrotisches Knochenfragment mit granulozytärer Überlagerung sowie Fibrinkondensat mit Plattenepitheleinschlüssen.

\section{Fall 3}

Es wurde ein 50-jähriger Patient nach Langzeitbeatmung über ein dilatativ angelegtes Tracheostoma vorgestellt zur Entfernung der Trachealkanüle. Endoskopisch fand sich eine subglottische Stenose mit von ventral sich vorwölbendem Granulationsgewebe und mäßiger trachealer Instabilität ( $\bullet$ Abb. 2a).

Nach Dekanülierung und Verschluss des Tracheostomas trat ein ausgeprägter Stridor auf. Es erfolgte das Wiedereinlegen der Trachealkanüle und die Abtragung des Granulationsgewebes durch Kryoextraktion. Dazu wurde zusätzlich ein Tracheoskop subglottisch plaziert und das Granulationsgewebe in 5 Teilstücken entfernt ( Abb. 2b). Die Dauer der Prozedur betrug 15 Minuten. Eine Argon-Plasma-Koagulation zur Blutungsstillung war nicht notwendig. Nach erneuter Dekanülierung war der Patient klinisch unauffällig. Auf ein thermisches Verfahren zur Gewebeabtragung suprastomal wurde verzichtet, um das Risiko einer

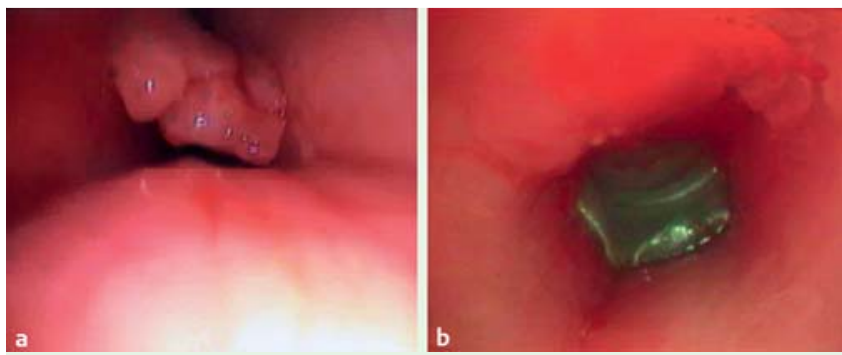

Abb. 2 a Endoskopischer Blick auf die subglottische Region mit Bildung von Granulationsgewebe. Die einliegende Trachealkanüle ist nicht sichtbar. b Identische Einstellung nach Kryoextraktion. Das Granulationsgewebe wurde komplett entfernt. Die Trachealkanüle ist wieder sichtbar.
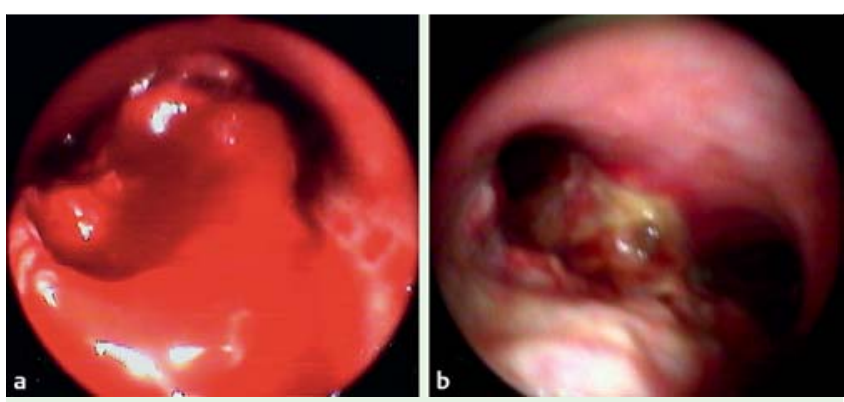

Abb. 3 a Blick auf die distale Trachea. Karzinosarkom mit Trachealstenose. Mit der Entfernung des Fremdgewebes wurde bereits begonnen. b Nach Rekanalisation noch flaches Tumorwachstum, vor allen Dingen an der Hauptbifurkation, in der dorsalen Trachea und im linken Hauptbronchus medial.

Trachealwandschädigung sowie einer Trachealkanülenbeschädigung zu minimieren.

\section{Fall 4}

$\nabla$

Ein 66 Jahre alter Patient kam zur stationären Aufnahme mit Ruhedyspnoe und radiologisch einer Totalatelektase links. Der bronchoskopische Befund erbrachte einen linksseitigen Hauptbronchusverschluss, eine hochgradige Trachealstenose des unteren Trachealdrittels ( $\mathbf{A b b} \mathbf{3} \mathbf{3}$ ) ) und eine hochgradige Hauptbronchusstenose rechts bis cranial des Abganges des rechten Oberlappenbronchus durch exophytisches Tumorwachstum.

Es wurden sowohl Zangen- als auch Kryobiopsate entnommen, sodann in der Kombination aus starrer und flexibler Bronchoskopie mit der flexiblen Sonde Kryoextraktionen durchgeführt. Die daraufhin erfolgte Computertomographie des Thorax ergab einen Normalbefund. Endoskopisch war lediglich noch flach exophytisches Tumorwachstum sichtbar ( Abb. 3b). Die Rekanalisation dauerte 35 Minuten. Es trat keine Blutung auf, die eine Argon-Plasma-Koagulation notwendig gemacht hätte. Die Zangenbiopsate erbrachten den Befund eines Lungenkarzinoms mit herdförmig neuroendokriner Differenzierung. Die Kryobiopsate ergaben den Befund eines Karzinosarkoms. 


\section{Diskussion}

Die vorliegenden Fälle zeigen für unterschiedliche Formen der Atemwegsverlegung die Möglichkeit der Rekanalisation mittels Kryoextraktion. Im Vergleich zum Laser besteht bei Gewebeentfernung nicht die Notwendigkeit einer clean-up Bronchoskopie, ohne Risiko können hohe Sauerstoffkonzentrationen verabreicht werden und die Gefahr einer Schädigung von Knorpel als bradytrophem Gewebe ist gering [1,2]. Darüber hinaus handelt es sich um ein sehr kostengünstiges Verfahren, das gesamte System ist für rund 7000 EUR zu erwerben. Ein Nachteil ist, dass die Kältesonde nicht mit dem anhaftenden Gewebestück durch den Arbeitskanal des Fiberskopes gezogen werden kann, weshalb ein Vorspiegeln über ein starres Bronchoskop oder einen Tubus notwendig ist.

Das Anhaften an der Kryosonde ist vom Wassergehalt der Zielstruktur abhängig [3]. Daher ist eine Extraktion von Mukus mit dieser Methode sehr leicht möglich. Fremdkörper aber können in der Regel nur entfernt werden, wenn ihnen durch eine entzündliche Reaktion Gewebe anhaftet.

Das Verfahren der Kryobiopsie liefert im Vergleich zur Zangenbiopsie relativ große Biopsate [4], zudem finden sich keine Quetschartefakte [5,6]. Hierdurch ist die gelungene histologische Diagnose eines pulmonalen Karzinosarkoms zu erklären. Denn normalerweise enthalten präoperative Biopsate dieses seltenen Tumors lediglich epitheliale Anteile [7].

\section{Interessenkonflikte}

Die Datenerhebung zu der in Lung 2009 publizierten Studie „Experimental study on biopsy sampling using new flexible cryoprobes: influence of activation time, probe size, tissue consistency, and contact pressure of the probe on the size of the biopsy specimen“ erfolgte in Zusammenarbeit zwischen Dr. Karl-Josef Franke, Dr. Georg Nilius und Professor Dr. Karl-Heinz Rühle mit Frau Dr. Szyrach und Herrn PD Dr. Enderle der Abteilung Forschung der Firma Erbe Elektromedizin $\mathrm{GmbH}$. Finanzielle oder Zuwendungen anderer Art fanden nicht statt.

\section{Literatur}

1 Hetzel M, Hetzel J, Schumann $C$ et al. Cryorecanalization: a new approach for the immediate management of acute airway obstruction. J Thorac Cardiovasc Surg 2004; 127: 1427-1431

2 Franke KJ, Nilus G, Rühle KH. Kryotherapie eines endobronchialen Lipoms. Pneumologie 2005; 59: 685-688

3 Homasson JP. Bronchoscopic cryotherapy. Journal Bronchol 1995; 2: $145-153$

4 Franke KJ, Szyrach M, Nilius G et al. Experimental study on biopsy sampling using new flexible cryoprobes: influence of activation time, probe size, tissue consistency, and contact pressure of the probe on the size of the biopsy specimen. Lung 2009; 187: 253-259

5 Hetzel J, Hetzel M, Hasel C et al. Old meets modern: the use of traditional cryoprobes in the age of molecular biology. Respiration 2008; 76: $193-197$

6 Babiak A, Hetzel J, Krishna G et al. Transbronchial cryobiopsy: a new tool for lung biopsies. Respiration 2009; 78: 203-208

7 Huwer H, Kalweit G, Straub U et al. Pulmonary carcinosarcoma: diagnostic problems and determinants of the prognosis. Eur J Cardio-thorac Surg 1996; 10: $403-407$ 